\title{
Category Theory as Interpretation Law Model in Artificial Intelligence Era
}

\author{
Lambrini Seremeti $^{1,2}$, Ioannis Kougias ${ }^{2,}$ * \\ ${ }^{1}$ School of Education, Frederick University, Nicosia, Cyprus \\ Email: lamprini.seremeti@gmail.com \\ ${ }^{2}$ Laboratory of Interdisciplinary Semantic Interconnected Symbiotic Education Environments, \\ Electrical and Computer Engineering Department, \\ University of Peloponnese, Peloponnese, Greece \\ Email: kougias@uop.gr \\ *Corresponding Author: Ioannis Kougias, Email: kougias@uop.gr
}

How to cite this paper: Lambrini Seremeti, Ioannis Kougias (2021). Category Theory as Interpretation Law Model in Artificial Intelligence Era. Journal of Artificial Intelligence and Systems, 3, 35-47. https://doi.org/10.33969/AIS.2021.31003

Received: January 11, 2021

Accepted: February 8, 2021

Published: February 9, 2021

Copyright (C) 2021 by author(s) and Institute of Electronics and Computer. This work is licensed under the Creative Commons Attribution International License (CC BY 4.0).

http://creativecommons.org/licenses/by/4.0/

\section{Abstract}

Nowadays, artificial intelligence entities operate autonomously and they actively participate in everyday social activities. At a macro-perspective, they play the role of mediator between people and their actions, as components of the fundamental structure of every social activity. At a micro-perspective, they can be considered as fixed critical points whose hypostasis is not subject to established legal framework. A key point is that embedding artificial intelligence entities in everyday activities may maximize legal uncertainty both at the macro and micro-level, as well as at the interim phase, i.e., the switch between the two levels. In this paper, we adapt a well-known concept from Category Theory, namely that of the pushout, in order to approximate the core interpretation legal framework of such activities, by considering each level as an open system. The purpose of using Systems Theory in combination with Category Theory is to introduce a mathematical approach to uniquely interpret complex legal social activities and to show that this novel area of artificially enhanced activities is of prime and practical importance and significance to law and computer science practitioners.

\section{Keywords}

Artificial Intelligence entities, Category Theory, legal social activity, macro-level, micro-level, pushout structure, Systems Theory.

\section{Introduction}

New human actions such as traveling by car, where the driver instead of holding the steering wheel is watching his/her favorite movie, or walking in the park while our electronics assistant is ordering the weekly shopping from the supermarket, or to be doing our morning exercise and at the same time informing our personal physician 
of our blood pressure are some of the daily activities, which are now easily realizable thanks to the technological developments, especially those which make use of intelligent systems.

Given that intelligent systems have plasticity and flexibility, their ability of learning and remembering even under insufficient information, their multilevel organization and their effective management of ambiguity and partiality, allows us to consider them as discrete entities with an active action in the natural environment. The question that arises is, who is responsible for any deviation from the original plan for the smooth implementation of such an activity?

In this effort to change, reform or innovate reality, what parameters should be studied in order to ensure the coherence of social becoming, the smooth transition from a lawful action in a known natural environment to a lawful action in an unknown artificial environment of high heterogeneity? What will be the appropriate correspondences and proportions between the two environments, so that the fundamental principles of lawful action are maintained? How are the problematic situations that are determined by the action of the individual elements of these environments delimited and solved?

The need for an interpretive model arises since, now more than ever, we are facing these open, complex problems. Complicated issues that can be interpreted in many ways are considered to be open and can therefore accept different solutions, depending on the filters that one applies in order to fit them into a known problematic situation. Complex are the problems that describe systems of great complexity, high variability and with varied interactions between their parts.

Beyond the terms, the proportions, the correspondences and the parameters, for the description of an organized complexity there is the meaning of the system, as it is derived from the Systemic approach, which refers to the system as a unity of interdependent and interacting individual elements which are organized in such a way so as to serve a specific purpose.

In this article, the application of Category Theory is studied as the mathematical model of interpreting activities in artificial intelligence environments, through the systemic view, as this is an interdisciplinary approach to describe the operation and behavior of highly complex systems. Next, the problem is delimited and the main features that justify the choice of the systemic view of Category Theory as an interpretive model of the social system, after the assimilation of the intelligent artificial entities, adapted to the conceptual map of the given problem are set. In paragraph 3 the theoretical background is briefly presented, whereas in paragraph 4, the proposed approach is visualized through an example. Section 5 consists of a thorough discussion, as well as our future work, and, finally, the concluding 
remarks are given in section 6 .

\section{The Problem}

The transition of social cohesion from a known natural anthropocentric environment to an unknown one of high heterogeneity that also incorporates qualitative characteristics of artificial intelligence entities, carries risks, mainly legal activities ensured through the principles and rules of law that govern spatio-temporally every society.

Nowadays, any social activity of commercial or recreational nature involves the use of automated decision-making technology. These are artificial entities (AE) that use plethora of data and specialized learning algorithms in order to achieve a specific goal [5], [14], [23]. Given that these AE cooperate with the human factor to carry out any number of different activities, the Parliaments of all Countries seek the legal framework of lawful responsibility in case of deviation from the provisions of the code of conduct [8], [9], [10]. Which behavior is in question though? That of the artificial intelligence entity or that of the human factor that created the AE? Can the $\mathrm{AE}$ be subject to a body of rights and obligations, since it has an initiative? But does it have a sense of entitlement to rights? Does it have a sense of remorse, in case it is to be punished for any caused damage? Is the human factor responsible? But if he/she designed it to achieve a different goal and yet the algorithm, taught from the environment, has changed the implementation of that goal? What of the human factor who just used the AE? And what if he/she was unaware of the internal structure and operation of the AE? Despite the fact that relevant concerns have been expressed, based on the international literature, the above questions have not been sufficiently answered [2], [18], [25], [32], [39].

In the light of this questioning, the coexistence of natural and artificial entities in a spatio-temporal dimension of high complexity, where each of the entities comes from a system of different characteristics seems to be a necessity. Physical entities are governed by a predetermined anthropocentric system of principles and rules, while artificial entities are governed by the principle of purpose orientation, and as they have the ability to learn from a plethora of data, they develop their own system of rules of operation.

Looking at the problem in parts, it is obvious that these are two discrete categories. The category of previously legally delimited entities (natural, person, legal entity, product) and the category of artificial non-legally defined entities (algorithm, robot). Each of these categories is a set of elements endowed with a certain set of relationships with each other, so as to form a whole that interacts with its external environment. Furthermore, each one is characterized by its internal distinct 
structure and the external behavior that they manifest. However, these are also the characteristics of the system in the light of Systemic Theory, which attempts to interpret entireties of great complexity.

Looking at the problem holistically, it is obvious that the smooth interaction of these two categories and their merging into a new category is required, which should incorporate the rules and principles of the previous two, so as to maintain coherence in the relationships between the elements of each category as well as the creation of new relations between the heterogeneous elements of the previous two categories. This merging of the involved categories though can rationally and strictly be described using the mantle of Category Theory.

\section{Theoretical Background}

By focusing on Artificial Intelligence (AI) applications, one finds that the definition depends on the framework of reference. From a research point of view, it is defined as 'the computational part of the ability to achieve goals in the world' [22]. From a computer science perspective, it can be defined as 'a branch of computer science that studies the properties of intelligence by synthesizing intelligence' [31]. In that sense, AI involves plenty of techniques, such as machine learning, probabilistic reasoning, logic programming, fuzzy logic, ontology engineering. Thus, defining AI is a complex task. It is therefore even more difficult to define AI systems or AI applications from a regulatory perspective, due to their high level of complexity. Regulation cannot be AI neutral since it aims at governing the AI embedded social activities. It must be evolved in accordance with technological advances in order to monitor the impact of AI on individual and social rights. Given that those so diverse fields, i.e., technology and law, are today separately treated, we propose Systems Theory to represent the complexity of AI applications and Category Theory to sketch their interpretation in the light of regulation.

To describe an organized complexity there is the concept of system, as it is derived from the Systematic approach which refers to the system as a set of interdependent and interacting individual elements that are organized in such a way as to serve a specific purpose [3].

A system is considered to be an organized set of components that interact, so that this set exists, is maintained and mutates in relation to a wider external environment. In interacting with the environment, the system's components use functions of input transformation (any information that enters the system from the external environment and changes its structure and operation) to outputs (any system behavior that is detected in the external environment).

The Systemic approach is distinguished by characteristics such as capacity, holism, 
Lambrini Seremeti et al.

syntheticity, based on which it defines the meaning of system. Category Theory, possessing same features, is its corresponding mathematical analogue [16]. When one is referring to the concept of system in the Systemic approach, it equivalently corresponds to the concept of category from the Category Theory point of view and the components of the system are respectively the objects of each category. The relationships between the components of the system correspond to the morphisms between the objects of the category. The interaction of the system with the environment corresponds to the functors between the categories. All the above hold, depending on the level of analysis of the system. If we refer to each component of the system as a distinct system with its own structure and function, then respectively in Category Theory, we can define different categories and functors. Therefore, Category Theory provides us with the possibility of a multilevel interpretation of a whole.

Category Theory is an alternative mathematical view for interpreting the correlations between heterogeneous objects that create the idea of an organized whole. It is governed by rules that emphasize not the objects themselves but the relationships between them. It also has the dynamic nature required to interpret an ever-changing system, as it focuses on how to move from one category to another, while maintaining their internal structures. It includes appropriate mathematical constructions, based on which it is possible to study dynamic processes [17].

From a philosophical point of view, Category Theory eliminates the objects/entities of categories that are abstractly defined and deals only with processes, i.e., the ways of moving from one object to another or from one category to another, while maintaining their internal structure. The basic idea behind using Category Theory as a mathematical tool for interpretation is that it is not concerned with the truth or falsity of a proposition, rather than with how that proposition relates to other propositions [11]. In mathematical language, it uses morphisms, functors, and natural transformations through which it describes the ways in which different structures are maintained or resembled, without trying reduction to specific entities. It mainly focuses on changing, not constant but isomorphic objects, through categorization. Another key advantage of using Category Theory as an interpretive model is the visualization of processes through diagrams. The diagrams follow the basic principles of this mathematical theory, which are based on objects, relationships between them, as well as compositions between relationships.

More specifically, in Category Theory the basic structural elements are the categories (sets of objects that are connected to each other by relations - i.e., the morphisms), the functors between the categories (laws according to which each object of one category corresponds to an object of another category and each 
morphism of one corresponds to one morphism of the other) and the natural transformations (laws that connect functors to each other). Category Theory also uses a set of universal constructs with which it is possible to highlight the relationships between seemingly distinct concepts in different categories, as it is the pushout.

In the international literature, the use of a mathematical theory in conjunction with legal science is mainly concerned with the standardization of legal reasoning through Mathematical Logic [4] [12], [26], [34], in designing dispute resolution strategies through Probability Theory [1], [7], [27], and visualizing the principles of the influx of crimes through Set Theory [24], [38]. Category Theory has been used as a mathematical tool in legal science solely because of the multi-layered analysis of a concept, its visualization by using diagrams and the ability of transition from one level of analysis to another [13], [33]. In this work we try, without going deeper into the strict language of Category Theory, to apply, using an example, a category-theoretical construction that ensures the one-to-one interpretation of a social activity in a heterogeneous environment.

\section{Merging to a single set of rules}

In the real world, every human activity involves persons and things that are legally qualified and for which there is a set of rules that govern any contractual relationship between them. This relationship is determined by the discrete role of each physical entity in the framework of a particular activity. For example, in a buying and selling activity, there is a corresponding legal framework that defines the rights and obligations of the parties, depending on their role as either seller or buyer. In a family relationship, each participant has a different role, thus a different institutional framework in which he/she belongs. In an imaginary world of absolute artificial intelligence, there will be similar contractual relationships between software agents that will be governed by a corresponding set of rules in resolving disputes. For the smooth transition from the current real world to this imaginary one, where people will again be the dominant body of rights and obligations, a special way of integrating the rules of operation of intelligent systems into the already institutionalized anthropocentric law is required. The smooth integration of one category into another can be done through the mathematical construction of the pushout, which conceptually corresponds to a merging of the two categories. 


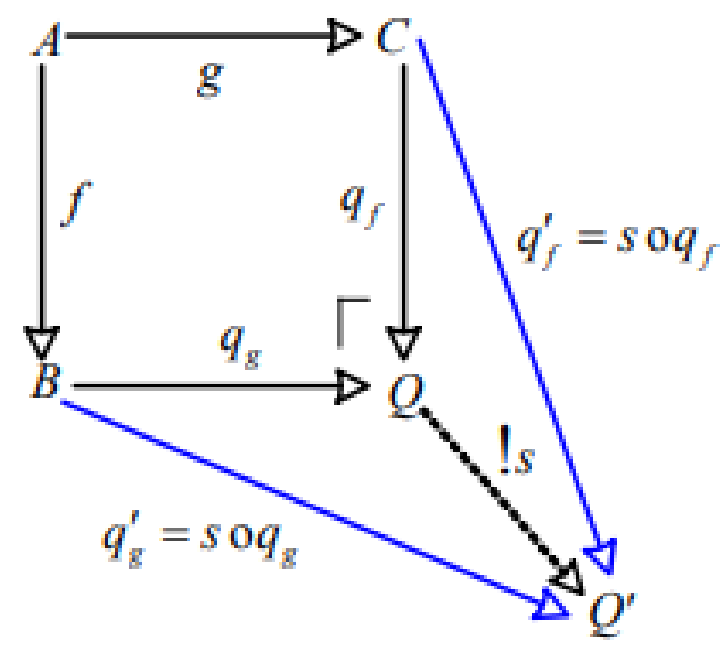

Figure 1. The pushout constructor of Category Theory

In the mathematical language of Category Theory, a pushout (Figure 1) is a triad $(Q, f, g)$ which ensures that the object $Q$ is also the only object that can incorporate the conceptual content of objects $B$ and $C$, since any other object $Q^{\prime}$ that will be created, it is bound to be isomorphic to $Q$. All these apply at the micro-level, between objects of a category.

In Figure 1 , the arrows $f, g, q_{f}, q_{g}$ are called morphisms and for each morphism there is an object at the blunt end of the arrow called domain and an object at the sharp end of the arrow called codomain. The morphisms $q_{g}^{\prime}=s \circ q_{g}$ and $q_{f}^{\prime}=s \circ q_{f}$ are called compositions of the corresponding morphisms, which means that the relative arrows share the same objects, where the domain of one arrow is the codomain of the other one. This means that the square diagram commutes. In the commuting square diagram, there are three different codomains that determine three different types of interpretation and thus the context of decision making in a specific problem is formed. The span $C \leftarrow f-A-g \rightarrow B$ is called projection and it can be interpreted as the maximization of uncertainty, since there appears to be no linkage between $B$ and $C$, while the span $B-q_{f} \rightarrow Q \leftarrow g_{f}-C$ is called injection and it can be interpreted as the maximization of certainty, since the two morphisms share the same codomain. The unique $s$ morphism is called m-morphism which is an ideal link directly from $Q$ to $Q^{\prime}$, that is, there is an isomorphism between the two objects $Q$ and $Q^{\prime}$. This isomorphism enables one to determine the identity and uniqueness of entities in terms of their relations to each other. From a philosophical perspective, a pushout is 
a teleological system at a micro-level, that is a result-oriented mathematical tool. With the process of merging, a new general category emerges that keeps the structural elements of the two previous categories unchanged, while, at the same time, the necessary relations are created between the objects of the two categories, without including unnecessary information.

In order for the proposed approach to be better understood, we give a simple example. Assume, at the micro-level, that the category $C_{1}$ conceptually corresponds to a sales activity. The objects of the category are the contracting parties i.e., the seller, the buyer, as well as the product. The morphisms between the objects of the category reflect the relations between them, from a legal point of view, namely through the rules of the law of obligation. Moreover, each object exists legally exclusively and only with reference to the relations that have been formed with the other objects of the same category. With the appropriate composition of the morphisms, the legal reasonings that apply in the specific case are realized. Category $C_{2}$ conceptually corresponds to the relationships between the components of an intelligent product, which initially exist but do not comply with the rules of law established to date. At the phase of switching from the micro-to the macro-level, the functors are the main category theoretic tools. A functor is defined as a morphism between two categories where each and every object and morphism are preserved, but are not necessarily the same. The functors $F 1,3$ and $F 2,3$ assign each object of the previous categories to an object of the new category $C_{3}$, maintaining the structure of the previous categories but adding new relationships through the synthesis of pre-existing morphisms. This entire diagram represents the merging of two categories into a new category, by the pushout, incorporating the objects of category $C_{2}$ into the structure of category $C_{1}$ in such a way as to create only the necessary new correlations, without redundancies. All this hold true, considering the elements of the diagram of Figure 2 , as objects of a wider category which will include the whole set of activities that can be implemented under the existing law.

Thus, at the macro-level, we can think of all the categories $C_{1}, C_{2}, C_{3}$ as objects of another generalized category representing all the social activities as morphisms between established law frameworks. Thus, in terms of Category Theory, the diagram of Figure 2 may be considered as the analogous of the pushout of Figure 1, tracing back at the micro-level. In that sense, in Figure 2, every category satisfies all the standard elementary conditions of a category, i.e., definition of objects and morphisms and axioms of the identity and association. In the proposed model $F 1,3$ and $F 2,3$, at the macro-level, allow comparisons between the corresponding structures. These regulatory functors create bridges between heterogeneous 
categories. Alternatively, viewing Figure 2, at the micro-level, the structure $C_{1} \rightarrow C_{3} \leftarrow C_{2}$ is the pushout of Figure 1, that is the cospan of injections $-F 1,3 \rightarrow C_{3} \leftarrow F 2,3-$ will be isomorphic to every other structure similar to $C_{3}$.

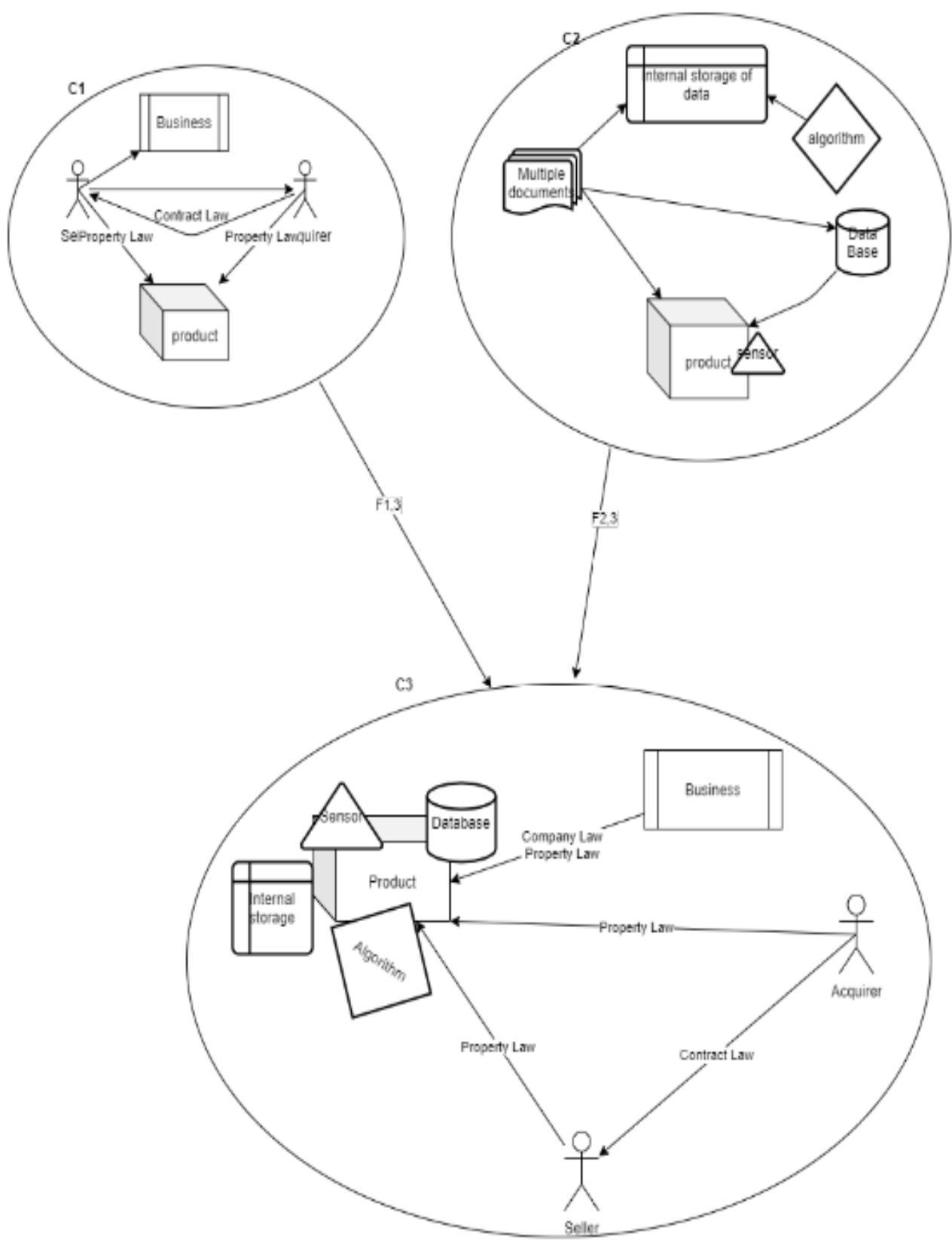

Figure 2. Merging of two Categories concerning a purchase and sale activity 
At this point, we emphasize that finding a new category through the pushout mechanism is only possible in cases where there are overlapping elements between the two original distinct categories. Otherwise, it may not be possible to find new relationships between independent elements, no new category can be formed.

\section{Discussion and Future Work}

Artificial Intelligence advances in combination with Law have attracted a lot of attention recently. A number of research articles have been published, from reasoning and knowledge representation to interpretation [6], [19], [27], [28], [29], [30], [36], [37]. Interpretation of law takes place whenever the meaning of a legal document has to be assessed in a concrete situation [35]. On the other hand, mathematical modelling is the process of translating beliefs about how a system functions into concise and precise language in order to identify underlying assumptions [29]. It is especially used for developing scientific understanding of organizational structures within the system being modeled and the mechanisms through which changes occur. An attempt to include in a model all aspects of a real-world system, by representing them in the form of equations, is inappropriate or inadequate [21]. Hence, there is a need for more theoretical models.

This article does not suggest a mathematical model based on the analysis of input and control data of a process in order to predict the output parameters of a law system [15], [16], but the possibility of developing a theoretical model that can pave the way for a legal interpretation process in a dynamic environment of experts (computer scientists, mathematicians, legal professionals). The proposed approach, using notations from Category Theory and the Systemic Approach, suggests a model which is a conceptual schema consisted of elements, relations, operations and rules governing interactions. Its importance as well as its novelty lie upon the fact that it provides a starting point for a deeper understanding of the definition of legal social activities, since its pictorial representation tends to communicate the key premises of the model to non-specialists in mathematical relations and operations.

As future work, we plan to enrich the proposed theoretical model with semantics, by using legal ontologies, in order to deal with inconsistencies emerging from legal reasoning.

\section{Conclusions}

This article attempts to highlight the complex problems occurred during the transition of social cohesion from a natural environment to an unpredictable environment in which autonomous AI entities are embedded. After portraying 
Lambrini Seremeti et al.

several features of AI entities which make them act in an unforeseeable way, it suggests an interpretation law model based on Category Theory.

With the proposed approach we ensure the uniqueness of the interpretation of any social activity in an environment in which the action of natural and artificial entities is involved, since the mathematical construction of the pushout ensures that all the categories created by merging two previous ones will be isomorphic, in other words they contain the same entities and the same correlations between them. Thus, the way of interpreting the rules of law that apply between the entities of the new categories are univocally defined.

\section{Conflicts of Interest}

The authors declare that there is no conflict of interest.

\section{References}

[1] Aaron, M.C. (2019) Risk and Rigor: A lawyer's guide to decision trees for assessing cases and advising clients, DRI Press, USA.

[2] Barfield, W. (2018) Liability for autonomous and artificially intelligent robots. Journal of Behavioral Robotics, 193-203. https://doi.org/10.1515/pjbr-2018-0018

[3] Bertalanffy, L. (2003) General System Theory: Foundations, Development, Applications. Revised Edition, Penguin University Books, New York.

[4] Brasil, S.M. and Garcia, B.B. (2003) Modelling legal reasoning in a mathematical environment through model-theretic semantics. Proceedings of the 9th International Conference on Artificial Intelligence and Law, Association for Computing Machinery, New York, United States, 195-203.

[5] Chakraborty, A. and Kar, A.K. (2017) Swarm intelligence: A review of algorithms. In: Patnaik, S., Yang, X-S, and Nakamatsu, K., Eds, Nature-Inspired Computing and Optimization. Theory and Applications, Springer International Publishing, 475-494.

[6] Chesterman, S. (2020) Artificial intelligence and the limits of legal personality. International and Comparative Law Quarterly, 69(4), 819-844, https://doi.org/10.1017/S0020589320000366

[7] Davies, S. (2009) Proof on the balance of probabilities: What this means in practice. https://uk.practicallaw.thomsonreuters.com/2-500-6576?transitionTy pe=Default\&contextData=(sc.Default)

[8] EPRS, PE 624.261 (2019) Understanding algorithmic decision-making: Opportunities and challenges. https://www.europarl.europa.eu/thinktank/en/document.html?refer ence=EPRS_STU\%282019\%29624261

[9] EP, European Parliament (2017) Resolution (2015/2013 (INL)). https://eur-lex.europa.eu/legal-content/EN/TXT/?uri=CELEX\%3A520 17IP0051

[10] EP, European Parliament (2020) Artificial Intelligence and Civil 
Liability. https://www.europarl.europa.eu/RegData/etudes/STUD/2020/62192 6/IPOL_STU(2020)621926_EN.pdf

[11] Girard, J. (1995) Linear Logic: Its syntax and semantics. In: Girard J., Lafont, Y. and Regnier, L., Eds., Advances in Linear Logic, Cambridge University Press, 1-42.

[12] Goldberg, S.P. (1981) On legal and mathematical reasoning. Jurimetrics, 22(1), 83-91, http://www.jstor.org/stable/29761772

[13] Heather, M. and Rossiter, N. (2017) What is Law? The perception of Category Theory. In: Beziau, J-Y., Buchsbaum, A. and Rey, C., Eds, Handbook of the 6th World Congress on Universal Logic, Publications of University of Vichy, France, 560-561.

[14] Kallen, S.R. (2012) Artificial intelligence algorithms. Journal of Computer Engineering, 6(3), 1-8.

[15] Kaye, D.H. (1980) Mathematical models and legal realities: Some comments on the Poisson model of jury behavior. Connecticut Law Review, 13(1), 1-17.

[16] Keon, R. (1980) Mathematical models for legal prediction. Computer/Law Journal, 2(1), 830-862, http://repository.jmls.edu/jitpl/vol2/iss1/29

[17] Lawvere, F.W and Schanuel, S.H. (2009) Conceptual Mathematics: A first introduction to categories, Second Edition, Cambridge University Press.

[18] Lion, A. (2020) AI entities as AI agents: Artificial Intelligence liability and the AI respondeat superior analogy, Mitchell Hamline Law Review, 1-58 https://ssrn.com/abstract=3446115

[19] Liu, H., Maas, M., Danaher, J., Scarcella, L., Lexer, M. and Van Rompaey, L. (2020) Artificial intelligence and legal disruption: A new model for analysis. Law, Innovation and Technology, 12(2), 205-258, https://doi.org/10.1080/17579961.2020.1815402

[20] Mac Lane, S. (1996) The development and prospects for Category Theory, Applied Categorical Structures, 4, 129-136. https://doi.org/10.1007/BF00122247

[21] Mazur, J.E. (2006) Mathematical models and the experimental analysis of behavior, Journal of the Experimental Analysis of Behavior, 85(2), 275-291. https://doi.org/10.1901/jeab.2006.65-05

[22] McCarthy, J. (2007) What is artificial intelligence? http://www-formal.stanford.edu/jmc/whatisai.pdf

[23] Mohammed, M., Khan, M.B. and Bashier, E. (2017) Machine learning: Algorithms and applications. International Standard Book, Number 13, Taylor \& Francis Group, LLC.

[24] Parker, B. (2017) Crisp-set qualitative comparative analysis (csQCA) and criminology. Journal of Qualitative Criminal Justice \& Criminology, 5(2), 153-173. https://eprints.qut.edu.au/114388/23/_qut.edu.au_documents_Staff Home_StaffGroupl\%24_lindequm_Desktop_ePrints_114388.pdf

[25] Ponkin, I.V. and Redkina, A.I. (2018) Artificial intelligence from the point of view of Law. Journal of Law, 22(1), 91-109. https://doi.org/10.22363/2313-2337-2018-22-1-91-109

[26] Potts, H., (1913) An application of Mathematics to Law. Nature 91, 
Lambrini Seremeti et al.

270. https://doi.org/10.1038/091270c0

[27] Rhee, R.J. (2006) A price theory of legal bargaining: An inquiry into the selection of settlement and litigation under uncertainty. http:// scholarship.law.ufl.edu/ facultypub/ 494

[28] Rissland, E.L. (1990) Artificial intelligence and law: Stepping stones to a model of legal reasoning. The Yale Law Journal, 99(8), 1957-1981, https://doi.org/10.2307/796679

[29] Scherer, M.U. (2016) Regulating artificial intelligence systems: Risks, challenges, competencies, and strategies. Harvard Journal of Law \& Technology, 29(2), 354-400.

[30] Scherer, M. (2019) Artificial intelligence and legal decision-making: The wide open? Journal of International Arbitration, 36(5), 539-573.

[31] Stone, P., Brooks, E., Brynjolfsson, R, Calo, O., Etzioni, G., Hager, J.,Hirschberg, S., Kalyanakrishnan, E., Kamar, S, Kraus, K., Leyton-Brown, D., Parkes, W., Press, A., Saxenian, J., Shah, M. and Teller, A. (2016), One hundred year study on artificial intelligence. https://ai100.stanford.edu/

[32] Surden, H. (2018) Artificial Intelligence and Law: An overview. https://readingroom.law.gsu.edu/gsulr/vol35/iss4/8

[33] Tanega, J.A. (2013) Default invariance: A naïve category theory of Law and Finance. In: O’Sullivan, P., Ed., Hubris to Disgrace: The Philosophy, Politics and Economics of Finance in the early 21 Century, Routledge, CRC Press, 1-82.

[34] Termini, M. (2019) Proving the point: Connections between legal and mathematical

reasoning, https://brooklynworks.brooklaw.edu/cgi/viewcontent.cgi?article=20 67\&context=faculty

[35] Thombre, S.P. (2019) General principles of statutory interpretation with special reference to golden rule \& mischief rule. Journal of Law, 5(6), 135-140.

[36] Verheij, B. (2020) Artificial intelligence and law. Artificial Intelligence Law, 28, 181-206, https://doi.org/10.1007/s10506-020-09266-0

[37] Viola, L. (2018) Interpretation of the Law through Mathematical Models. Diritto Avanzato Edizioni.

[38] Voss, H.L. (1969) Differential association and containment theory: A theoretical convergence. Journal of Social Forces, 47(4), 381-391. https://doi.org/10.2307/2574526

[39] Zevenbergen, B., Finlayson, M.A., Kortz, M., Pagallo, U., Borg, J.S. and Zapusek, T. (2018) Appropriateness and feasibility of legal personhood for AI systems. Proceedings of the International Conference on Robot Ethics and Standards, CLAWAR Association Ltd., 1-8. 